\title{
Photostability of xanthene molecules trapped in poly(vinyl alcohol) (PVA) matrices
}

\author{
M. Talhavini, T.D.Z. Atvars* \\ Departamento de Físico-Química, Instituto de Química, Unicamp, Caixa Postal 6154, CEP 13083-970, Campinas, SP, Brazil
}

Received 8 May 1998; received in revised form 16 September 1998; accepted 20 September 1998

\begin{abstract}
Photobleaching reactions of fluorescein and Rose Bengal molecules dissolved in PVA matrices were studied as a function of the temperature. We used and compared two theoretical approaches to describe these photobleaching kinetics. Photobleaching quantum yield calculations were done using an adapted model (B-model) for polychromatic excitation that allowed us to estimate the average number of photons absorbed by each dye molecule before its bleaching. The other model uses exponential functions allowing us to calculate rate constants and activation energy. A slower photobleaching process was obtained for Rose Bengal sorbed in PVA matrices compared with fluorescein. We also obtained that the Rose Bengal photobleaching process is always single-exponential kinetic independent of the polymer relaxation process. Nevertheless, fluorescein photobleaching reaction is bi-exponential process at higher concentrations and it is strongly dependent on the polymer relaxation processes. These results were interpreted in terms of the different efficiencies among the processes involving quenching of the lowest triplet state. (C) 1999 Elsevier Science S.A. All rights reserved.
\end{abstract}

Keywords: Fluorescein; Rose Bengal; Photobleaching; Poly(vinyl alcohol)

\section{Introduction}

The xanthene dyes molecules have been used in many scientific and technological applications like dyes for laser systems, solar energy accumulators, holographic recording, immunoassay analysis and others. Among the polymer properties as low cost, high processability, light transparency, chemical and thermal stabilities, polymers are also interesting media for nonlinear-optical devices, optical computing, and selective chemical reactions [1-4].

The bleaching of xanthene molecules when exposed to electromagnetic radiation is a well-known phenomenon. While photobleaching must be avoided or minimized in dye lasers and immunoassays, it is a necessary process for holographic image printer. The information related to the photobleaching mechanism and its temperature dependence is, therefore, important for both fundamental studies and technological applications [5-8].

The chemical mechanism of xanthene photobleaching is very complex [5-12]. Fluorescein irradiated by visible light is photoreduced and produces a di-hydro leuco-dye [9]. Halogenate fluorescein derivatives are also photoreduced by visible light producing photodehalogenate colorless

*Corresponding author. E-mail: tatvars@iqm.unicamp.br forms, depending on the excitation wavelengths. For example, if Rose Bengal is irradiated with green light a sequential removal of the iodine atoms of the xanthene moiety is observed while the chlorine atom elimination of the phenyl moiety is only produced if shorter wavelengths (blue-green) light is employed [10,11]. Several experimental results demonstrated that the lowest triplet state is the intermediate state of the photobleaching reactions of xanthene dyes $[2,5,8,9]$.

In recent works we demonstrated that fluorescein photobleaching reaction rate increases with the temperature increase and becomes faster at temperatures above the poly(vinyl alcohol) (PVA) glassy transition [12]. Moreover, the kinetic description of the bleaching process is strongly dependent on the dye concentration. We also demonstrated that a single-exponential photobleaching rate constant could describe the reaction kinetic at lower concentrate samples. However, for higher concentrate samples, we were only able to describe the photobleaching kinetic by a bi-exponential function that includes a faster bleaching process involving D-D interaction $[2,5,12]$. Nevertheless, the relative importance of the PVA medium for the reaction mechanism has not been well established $[1,12]$.

In general, the requirement for kinetic description using bi-exponential function for dyes in polymer matrices can be 
interpreted by the presence of, at least, two different types of sorption sites for the guest molecules [13-18]. It is also assumed that the guest molecules exhibit different photophysical and photochemical properties [13-18]. Moreover, the bi-exponential photobleaching behavior should also be present if more than one type of reaction mechanism is operative $[8,19]$. We assumed earlier that the reason for the presence of a single-exponential photobleaching of fluorescein in lower concentrate and a bi-exponential for higher concentrate PVA matrices resulted of a sequential occupation of sites with different dye-polymer interaction strengths. In this work we will study if this is a general behavior for xanthene dyes [12].

In the present work we are comparing the temperature and dye concentration effects upon the photobleaching kinetics of two xanthene dyes dissolved in PVA: fluorescein and Rose Bengal. These two xanthene dyes present very different triplet quantum yields due to the presence of inner heavy atoms [20] and we will compare the relative importance of the electronic triplet state population on the effectiveness of the photobleaching response. In addition, we are reporting the results for the temperature dependence of the photobleaching reactions induced by a xenon lamp irradiation at several temperatures. We also applied two theoretical kinetic treatments to match the experimental data: a mono-exponential decay model and a modified version of the model proposed by Dubois et al. [21] to calculate the reaction quantum yield (B-model). Some necessary modifications of the original B-model were presented allowing its application to photochemical reaction with polychromatic instead of monochromatic irradiation. Using both models we have computed the average number of absorbed photons by each dye molecule before it bleaches and we compared the photobleaching efficiencies for both xanthene dyes.

\section{Experimental}

Poly(vinyl alcohol) (PVA) (Aldrich Chemical) $M_{\mathrm{W}}=$ $124000 \mathrm{~g} \mathrm{~mol}^{-1}, \quad 87-89 \%$ hydrolyzed, was used as received, and some of its properties was described elsewhere [22,23].

Fluorescein (FL) and Rose Bengal (RB) (Aldrich Chemical, Analytical grade) were recrystallized from an ethanolic solution, dried under vacuum and stored in dark. The dyed-PVA films were prepared as described elsewhere $[22,23]$. The dye concentrations were defined as $0.1 \%$ and $0.01 \%$ in mass. All of the doped films have good optical transparency and their thickness range was ca. 58-60 $\mu \mathrm{m}$.

A diagram of the experimental setup is shown in Fig. 1 [24]. The photobleaching processes of the dyed films were performed selecting the wavelengths of a xenon arc lamp by a cassette of interference/cutoff filters, chopped at $23 \mathrm{~Hz}$ and focused onto the sample surface. A $5 \times 5 \mathrm{~mm}$ film slice was covered with an aluminum disk with a $1 \times 1 \mathrm{~mm}$ square hole that was placed in a vacuum sealed head cryo-system (APD

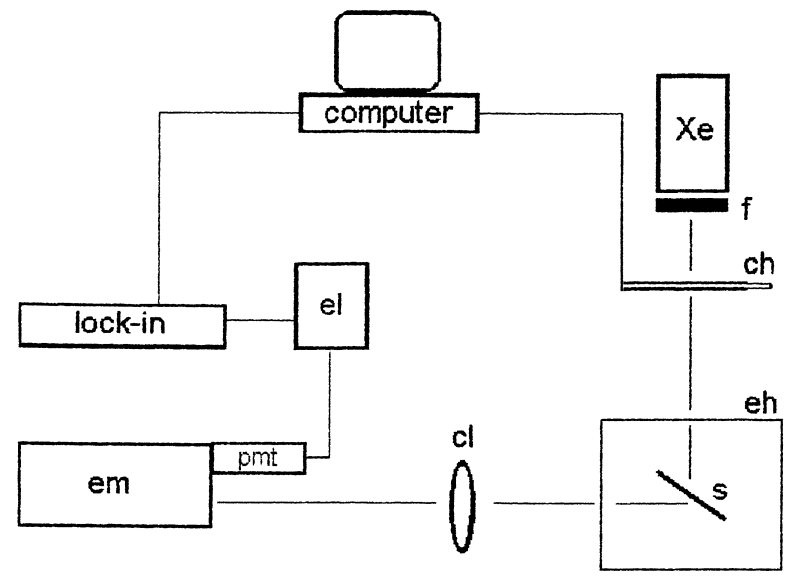

Fig. 1. Experimental setup including: Xe-150 W xenon lamp; f-filter and colimating lens; ch-chopper; eh-expansor head; s-sample; cl-cylindrical lens; em-Ebert monochromator; pmt-photomultiplier tube; el-electrometer.

Displex 204 system) equipped with Suprasil quartz windows. Since the light focus had a spot with $5 \mathrm{~mm}$ diameter, the radiant power that reached the sample was almost insensitive to its position. The sample fluorescence emission was collected from the back face and was focused by a cylindrical lens on the entrance slit of a $50 \mathrm{~cm}$ Ebert monochromator (Spex model 500M). The emission at selected wavelength was detected by an EMI 62565 PMT, the current generated was converted into potential difference by a Keithley (610C) electrometer and the signal was analyzed by a lock-in amplifier (Stanford Research SR530). A more detailed description of the instruments was reported elsewhere [24].

The photobleaching reaction was measured following the dye fluorescence intensity at $\lambda_{\mathrm{em}}(\mathrm{FL})=515 \mathrm{~nm}$ and $\lambda_{\mathrm{em}}$ $(\mathrm{RB})=590 \mathrm{~nm}$. The experimental data were collected at intervals of 5 or $10 \mathrm{~s}$ during a maximum of three hours, depending on the reaction rate. This procedure was repeated for several temperatures from 260 to $400 \mathrm{~K}$, which was selected by a digital temperature controller (Scientific Instruments, model 9650) with a precision of $\pm 0.5 \mathrm{~K}$.

\section{Theoretical background}

The experimental photobleaching curves were matched using two different theoretical approaches: (1) B-model, that was adapted from that developed by Dubois et al. [21] allowing us to estimate the photobleaching quantum yield and the reaction decay constant; (2) an exponential equation for the fluorescence intensity versus photo-reaction time allowing us to determine both the rate constants and activation energies. The goodness of the fit for both models was analyzed taking into account two parameters: the $\chi^{2}$ values and the distribution of residuals. We also compared the rate constants calculated using both models.

In this topic a summary of the analytical solutions for both models is presented. The B-model was developed for photo- 
chemical reactions induced by a monochromatic radiation [21] and for the numerical evaluation of the quantum yield it must be significantly modified. On the other hand, multiexponential functions were successfully applied to describe the photobleaching kinetic reaction of fluorescein in PVA matrix [12]. Since these two models generate different parameters, they could only be compared if specific equations for decay constants should be deduced. Therefore, we are presenting the background necessary to establish the modified B-model and how the calculated decay rate constants can be obtained and compared using both models.

\subsection{B-model}

Let us consider, using the same notation proposed by Dubois et al. [21], that under irradiation the dye- 1 molecule bleaches to another dye-2 molecule. We define the $z$-axis axis perpendicular to the sample surface with thickness, $L$, and parallel to the incident excitation beam. Let's also define the molecular density per area unity, $N_{1}(z, t)$ and $N_{2}(\mathrm{z}, \mathrm{t})$, as the population of dye-1 (unbleached) and dye-2 (bleached), respectively, which are dependent on both time and distance from the sample surface. Let's also consider $N_{0}$ as the total number of dye molecules present in the media. Then

$N_{1}(z, t)+N_{2}(z, t)=N_{0}$

The number of molecules of dye- 1 and dye- 2 along the sample thickness is

$$
\begin{aligned}
& J_{1}(z, t)=\int_{0}^{z} N_{1}(z, t) d z \\
& J_{2}(z, t)=\int_{0}^{z} N_{2}(z, t) d z
\end{aligned}
$$

and considering that $z$ is equal to the total thickness film $L$

$J_{1}(L, t)+J_{2}(L, t)=N_{0} L=J_{0}$

Considering that the photon flux through the sample thickness depends on its composition and that the photobleaching process is continuously occurring, we can write:

$n(z, t)=n_{0} \exp \left[-\Delta \sigma j_{1}(z, t)\right] \exp \left(-\sigma_{2} N_{0} z\right)$

where $n_{0}$ is the incident flux of photons with wavelength $\lambda$; $\sigma_{1}$ and $\sigma_{2}$ are the absorption cross sections, at $\lambda$, for unbleached and bleached molecules, respectively; and, $\Delta \sigma=\sigma_{1}-\sigma_{2}[21]$.

Defining $B$ as the average number of photons absorbed by a dye- 1 before it bleaches, the $B^{-1}$ is equivalent to the quantum yield for photobleaching reaction, and the local bleaching rate is [21]

$\frac{\partial N_{1}(z, t)}{\partial t}=-\sigma_{1} N_{1}(z, t) B^{-1} n(z, t)$

All of these parameters were described earlier.
Eq. (5) can be integrated taking into account that $J_{1}(L, 0)=J_{0}$, resulting

$\frac{J_{1}(L, t)}{J_{0}}=\frac{\ln \left[1+\exp \left(\Delta \sigma J_{0}-1\right) \exp \left(-\sigma_{1} B^{-1} n_{0} t\right)\right]}{\ln \left[1+\exp \left(\Delta \sigma J_{0}-1\right)\right]}$

To simplify the evaluation of $J_{1}(L, t)$ in Eq. (6) we considered two approximations: the sample is very thick and, then, the $z$ parameter could be considered independent of the photon flux, and, the dye-2 (bleached molecule) absorption coefficient at $\lambda_{\text {exc }}$ is virtually nil. Then, $\sigma_{2}=0$, and $\Delta \sigma=\sigma_{1}$ and Eq. (6) is re-written as:

$$
\begin{aligned}
I_{\mathrm{Fn}} & =\frac{I_{\mathrm{F}}(t)}{I_{\mathrm{F}}(0)}=\frac{J_{1}(L, T)}{J_{0}} \\
& =\frac{\ln \left[1+\exp \left(\sigma_{1} J_{0}-1\right) \exp \left(-\sigma_{1} B^{-1} n_{0} t\right)\right]}{\ln \left[1+\exp \left(\sigma_{1} J_{0}-1\right)\right]}
\end{aligned}
$$

where: $I_{\mathrm{Fn}}$ is the normalized fluorescence intensity calculated over the whole spectral range and $I_{\mathrm{F}}(t)$ and $I_{\mathrm{F}}(0)$ are the fluorescence intensities at time $t$ and $t=0$, respectively.

The evaluation of $B$ (the average number of photons absorbed by dye-1) assumes the knowledge of some parameters: the absorption cross section, $\sigma_{1}$, the initial flux of photons $J_{0}$; and the time-dependence of the dye concentration, which can be determined matching Eq. (7) to the experimental photo-reaction data.

As pointed out earlier, since the Eq. (7) is applied if monochromatic irradiation is used, the absorption cross section $\sigma_{1}$ is referred to one specific excitation wavelength. To estimate this value in experiments using polychromatic excitation source we established the following approach: (1) an excitation band is selected by a set of optical filters (Fig. 2(a)) coincident with the maximum absorption spectrum of fluorescein (Fig. 2(b)) or Rose Bengal in the visible region. (2) The integrated radiation intensity transmitted by the filter set was measured using a semiconductor radiometer, and the incident photon flux was calculated, $I_{0}=4.7 \times 10^{24}$ photon $\mathrm{s}^{-1} \mathrm{~m}^{-2}$. (3) The effectively absorbed photons for a specific wavelength range were supposed to be the product between the excitation intensity $I_{\lambda}$ (light source intensity) and $A_{\lambda}$ (sample absorption) and the resulted curve for fluorescein is presented in (Fig. 2(c)). This should be interpreted as a weight average absorption curve for unbleached dye and its integral is the average cross section value, $\sigma_{1}$, centered at the band maximum. (4) The wavelength at the intensity peak has been related to the maximum overlap between curves in (Fig. 2(a)) and (Fig. 2(b)) and is determined by

$$
\langle\lambda\rangle=\int_{0}^{\infty} \lambda f(\lambda) d \lambda
$$

integrated over the whole band. These average values are: $\langle\lambda\rangle=445 \mathrm{~nm}$ and $\langle\lambda\rangle=490 \mathrm{~nm}$ for fluorescein and Rose 

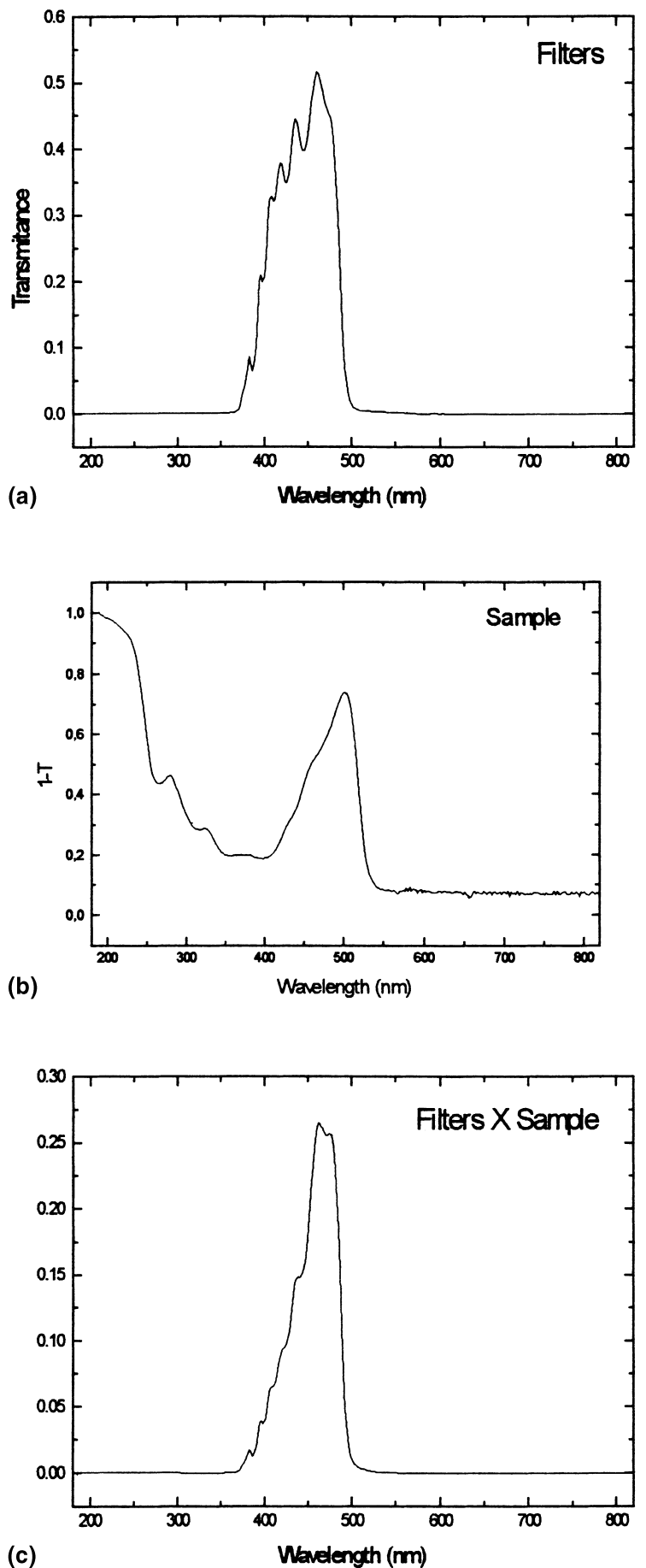

Fig. 2. (a) transmission window $(\mathrm{T}(\lambda))$ for the band pass filters used for Fluorescein excitation; (b) normalized absorption spectrum of $0.1 \% \mathrm{FL} /$ PVA (1-T); (c) plot of $\mathrm{T}(\lambda) \times(1-\mathrm{T})$.

Bengal, respectively. Therefore, using these procedure $\sigma_{1}$ (FL) $\left(\lambda_{\mathrm{abs}}=445 \mathrm{~nm}\right)=10.5 \mathrm{~m}^{2} / \mathrm{mol}$ and $\sigma_{1}(\mathrm{RB})\left(\lambda_{\mathrm{abs}}=\right.$ $490 \mathrm{~nm})=6.1 \mathrm{~m}^{2} / \mathrm{mol}$ were calculated.

Assuming that the dye concentration is very low to prevent bimolecular photochemical or photophysical processes and that the dye ability to absorb the excitation light is governed by the Beer's law, the fluorescence intensity will be proportional to the dye concentration in the electronic ground state. In the case of the present model the fluorescence intensity will be proportional to $J_{1}(L, t)$.

Finally, in an attempt to compare the two kinetic models we must define equations for the numerical evaluation of the decay constant. The equation for the decay constant can be derived assuming that the right side of Eq. (7) is equal to $1 / \mathrm{e}$ at the time $t_{1 / \mathrm{e}}$.

$t_{1 / \mathrm{e}}=\frac{-B \ln \omega}{\sigma_{1} n_{0}}$

where

$\omega=\frac{\exp (\gamma)-1}{\exp \left[\sigma_{1} j_{0}-1\right]}, \quad$ and $\gamma=\frac{\ln \left[1+\exp \left(\sigma_{1} j_{0}-1\right)\right]}{2.718}$.

\subsection{Exponential approach}

The exponential approach to describe kinetic processes occurring in polymer medium introduces a sum of two or more exponential functions to fit the experimental decay curves:

$I_{\mathrm{F}}(t)=C+\sum A_{\mathrm{i}} \frac{\exp (-t)}{\tau_{\mathrm{i}}}$

where: $I_{\mathrm{F}}(t)$ is the time-dependent fluorescence intensity, proportional to the electronic ground state population; $A_{\mathrm{i}}$ is a pre-exponential factor interpreted as an amplitude factor proportional to the relative contribution of every rate constant; $C$ is an integration constant; and $\tau_{\mathrm{i}}$ is a lifetime defined as the time necessary for the emission reaches to $1 / \mathrm{e}$ of the initial value.

We have shown earlier that a bi-exponential function is the best representation for the experimental data [12] of the photobleaching processes for $0.1 \%$ fluorescein in PVA at temperatures below the PVA glassy transition $\left(T_{\mathrm{g}}=350 \mathrm{~K}\right)$. Nevertheless, a single exponential function is able to give reasonable description of the photobleaching process for $0.01 \%$ fluorescein in PVA. These results were interpreted by the inhomogeneous distribution of the dye molecules average over, at least, two different types of guest sorption sites: one containing isolate molecules and other containing molecules separated by the Förster radium. Above $T_{\mathrm{g}}$, the polymeric matrix properties tend to be equalized and their time dependent processes, including photobleaching reactions, can be described by a single exponential function, such as in viscous media [13-15]. Therefore, the bi-exponential photobleaching decay was not only a more accurate model at higher concentrations but also demonstrated that a faster process involving triplet-triplet and triplet-ground state dye molecules interactions (D-D) was present $[8,12]$. Under the assumption that the experimental data should be represented by a mono-exponential function we could compute the decay constants from Eq. (10). 


\section{Results and discussion}

Experimental plots for the time dependence of the fluorescence intensity for both fluorescein and Rose Bengal in PVA films with two compositions $(0.01 \%$ and $0.1 \% \mathrm{w} / \mathrm{w})$ and several temperatures are shown in Fig. 3. The temperature range was chosen to cover values below and above the PVA glassy transition $\left(T_{\mathrm{g}}=345 \mathrm{~K}\right)[12,22,23]$. Comparing the experimental curves for both dyes, some important differences emerged: (1) the time scale for fluorescein/ pva photobleaching is shorter than that for Rose Bengal/ PVA revealing a faster photobleaching process; (2) since Rose Bengal/PVA bleaches with a slower rate, higher temperatures were necessary to a considerable dye consumption.

The experimental curves were fitted either by the B-model Eq. (7) or by exponential functions (Eq. (10)) for fluorescein and Rose Bengal in PVA at several temperatures. Although the mono-exponential function is very adequate to represent the kinetic behavior of Rose Bengal in PVA at both concentrations, only a bi-exponential function produces a better fit for fluorescein $0.01 \%$ in PVA. To perform a general data treatment applied for all samples we considered only single-exponential decay function in both cases Eq. (10).

Taking into account the experimental and simulated kinetic curves showed in Fig. 4, we observed that while
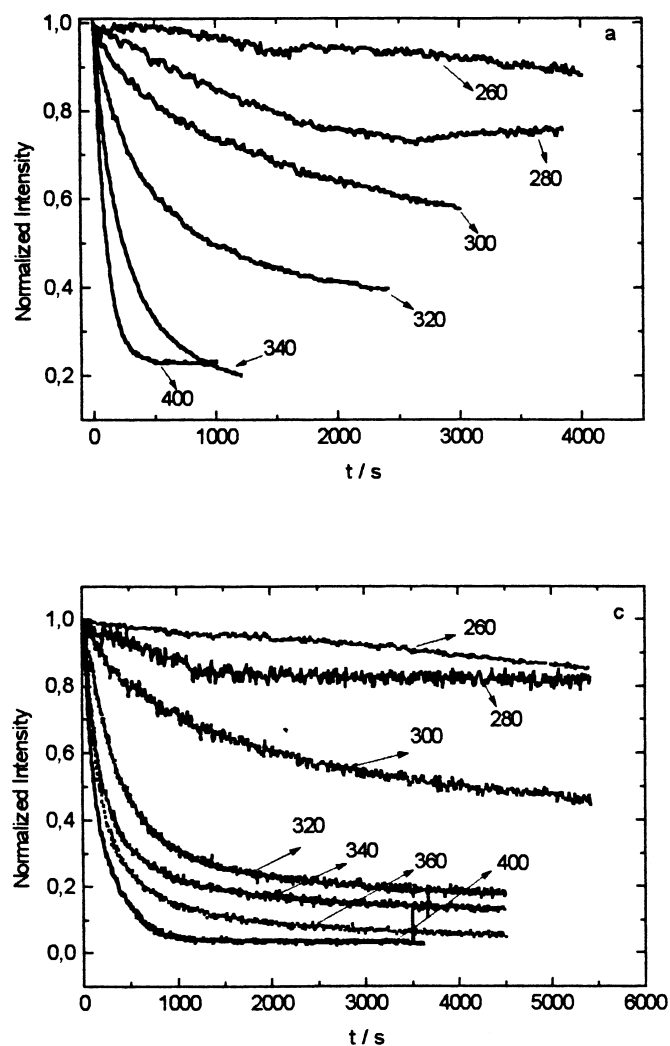

the mono-exponential functions provided a reasonable fit for all experimental data, B-model rendered a poor fit. We demonstrated earlier that the mono-exponential function was not the best function to match the experimental data for $0.1 \%$ fluorescein in PVA but this function provided a better fit than the B-model. Furthermore, the fit using the Bmodel was improved for temperatures higher than the PVA glassy transition.

The average number of photons absorbed by the unbleached dye ( $B$ values) was determined from the best fit of the experimental data using Eq. (7). Using Eq. (9) we calculate the photobleaching decay constants for every temperature was calculated and these values were compared with those from the exponential decay. Both set of values were listed in Table 1 for $0.1 \%$ FL/PVA and $0.1 \%$ RB/PVA samples. A comparison between these values shows: that the values determined using both models are within the same magnitude order; values for fluorescein are always lower than for Rose Bengal; there is a remarkable decrease of the decays at higher temperatures.Arrhenius plots for the temperature dependence of the photobleaching rate constants are presented in Fig. 5 using data obtained for mono-exponential functions. From these plots the apparent activation energies, $\quad E_{\mathrm{a}}(\mathrm{FL})=20.7 \mathrm{~kJ} \mathrm{~mol}^{-1}$ and $E_{\mathrm{a}}(\mathrm{RB})=85 \mathrm{~kJ}$ mol $^{-1}$, were calculated.

There also is a remarkable decrease (ca. three orders of magnitude) of decay constants for $0.1 \%$ FL/PVA between
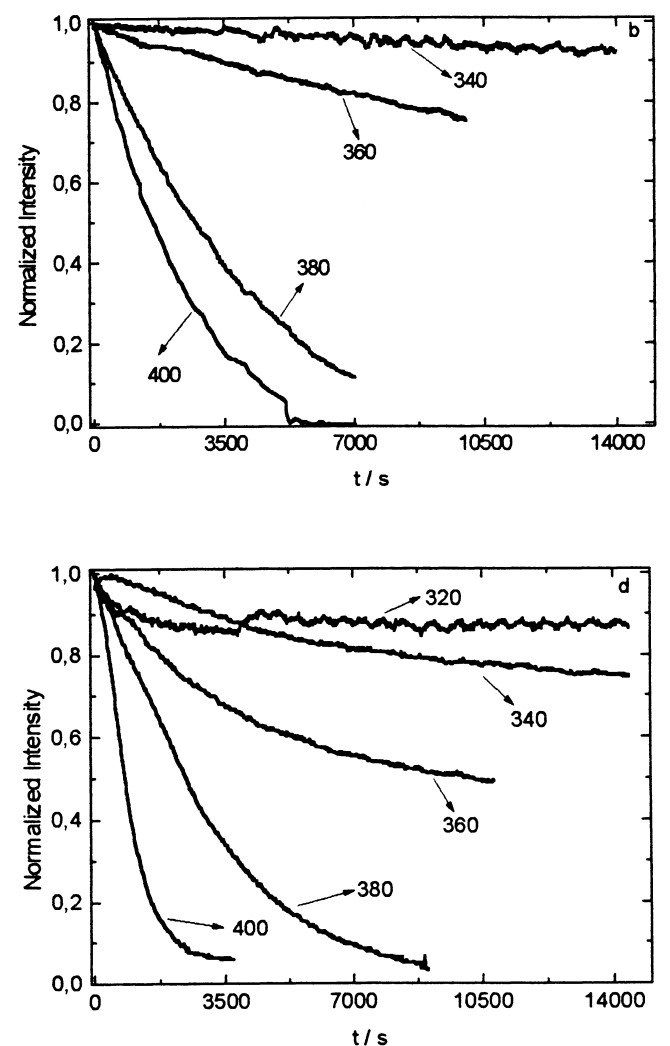

Fig. 3. Photobleaching curves for FL/PVA and RB/PVA samples at several temperatures and with compositions: (a) and (b) $0.01 \%$, and (c) and (d) $0.1 \% \lambda_{\text {em }}$ $(\mathrm{FL})=515 \mathrm{~nm}, \lambda_{\mathrm{em}}(\mathrm{RB})=590 \mathrm{~nm}$. 


$$
\mathrm{T}=280 \mathrm{~K}
$$
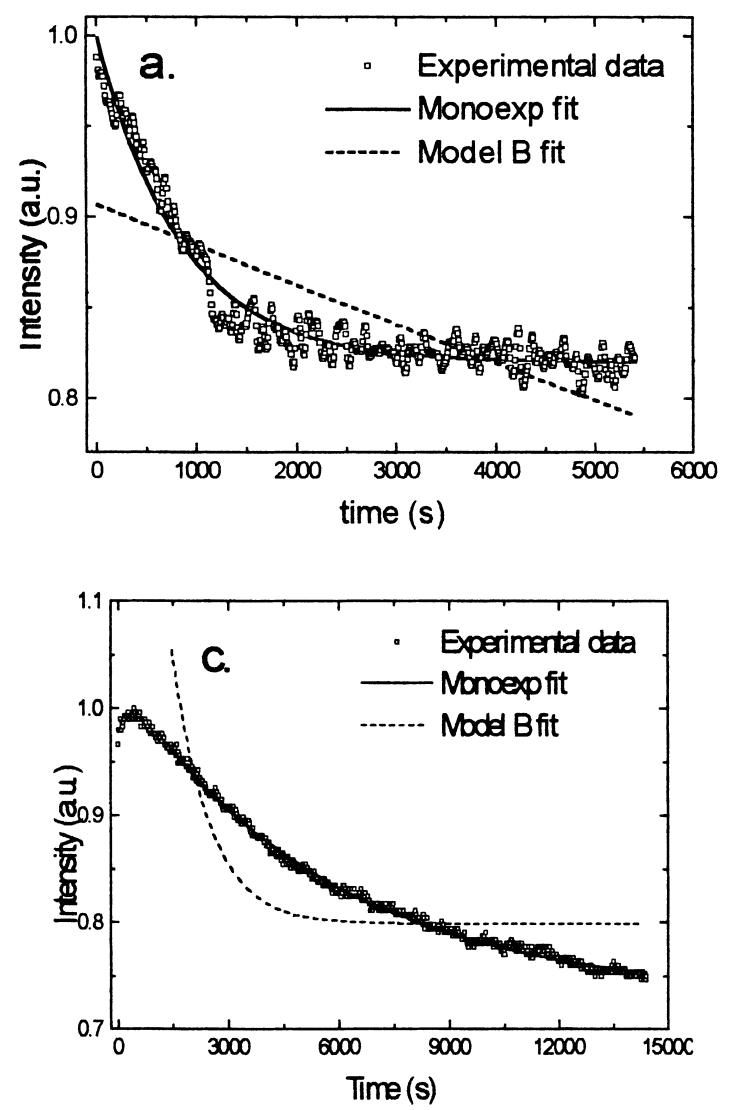

$\mathrm{T}=400 \mathrm{~K}$
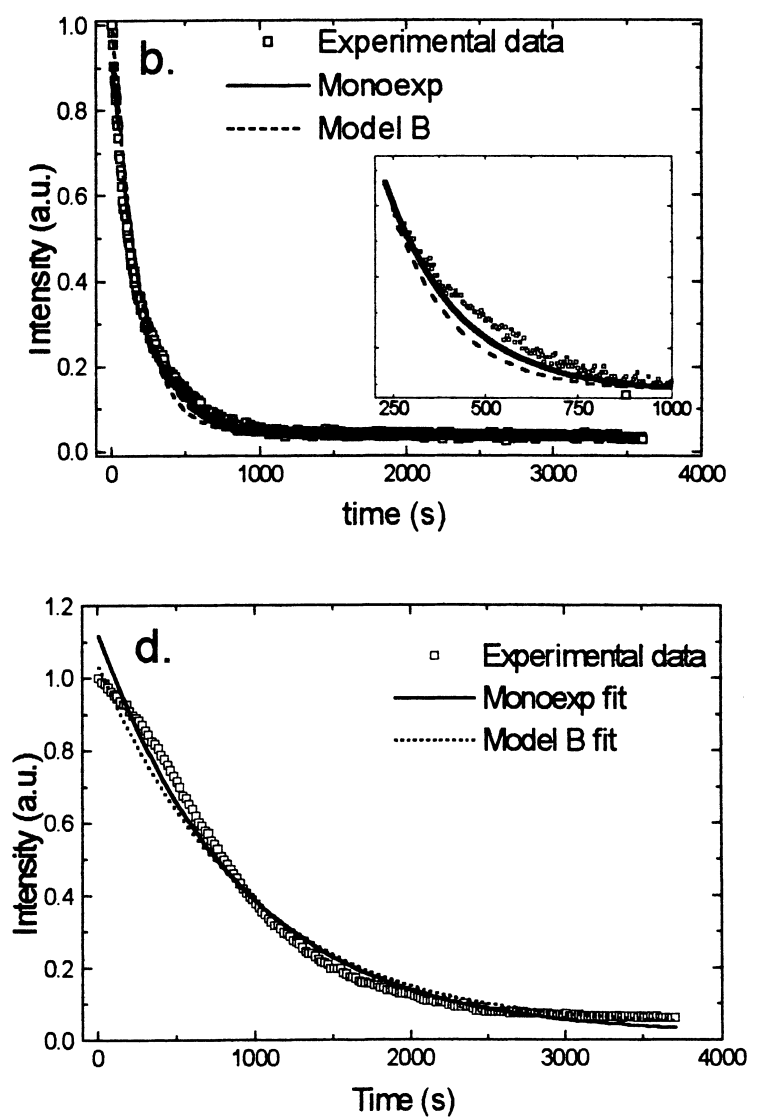

Fig. 4. Fluorescence intensity decay at $\lambda_{\mathrm{em}}(\mathrm{FL})=515 \mathrm{~nm}$ and $\lambda_{\mathrm{em}}(\mathrm{RB})=590 \mathrm{~nm}$ for $0.1 \%$ FL/PVA $((\mathrm{a}) T=280$ and $(\mathrm{b}) T=400 \mathrm{~K})$ and RB/PVA $((\mathrm{c})$ $T=280$ and (d) $400 \mathrm{~K}$ ) and samples: $\square$ experimental data, - - Eq. (7) and - Eq. (10).

260-280 K, calculated either by Eq. (9) (a poor adjustable model) or by Eq. (10) (a better kinetic model). Regardless that PVA undergoes relaxation processes in this temperature range ascribed to motions of molecular chains not hydrogen bonded $[25,26]$ we postulate that this relaxation processes induced important non-diffusional changes of the photobleaching reaction. However, this point should be studied in more detail.

In order to explain the remarkable differences between the fluorescein and Rose Bengal photobleaching rates in PVA, we considered a similar kinetic scheme for these reactions as proposed by Lindqvist $[27,28]$ and subsequently by Song et al. $[8,19]$ in their fluorescence microscopy studies. This scheme (reactions a-i) considered that the reactive state for xanthene dyes is the triplet excited state and several sequential photophysical and photochemical processes could take place (reactions $\mathrm{a}-\mathrm{i}$ ):

$$
\begin{aligned}
& \mathrm{T}^{*} \rightarrow \mathrm{S}\left(k_{1} \text { radiationless deactivation }\right) \\
& \mathrm{T}^{*}+\mathrm{T}^{*} \rightarrow \mathrm{T}^{*}+\mathrm{S}\left(k_{2} \text { triplet quenching }\right) \\
& \mathrm{T}^{*}+\mathrm{S} \rightarrow \mathrm{S}+\mathrm{S}\left(k_{3} \text { triplet quenching }\right)
\end{aligned}
$$

Table 1

Decay constants $\left(t_{1 / \mathrm{e}}\right)$ calculated by Eq. (9) and Eq. (10) for $0.1 \%$ FL/PVA and $0.1 \%$ RB/PVA samples.

\begin{tabular}{lllll}
\hline T/K & $t_{1 / \mathrm{e}} / \mathrm{s}$ (eq. 9) & $t_{1 / \mathrm{e}} / \mathrm{s}$ (eq. 9) & $t_{1 / \mathrm{e}} / \mathrm{s}$ (eq. 11) & $t_{1 / \mathrm{e}} / \mathrm{s}(\mathrm{eq} .11)$ \\
& $0.1 \%$ FL/PVA & $0.1 \%$ RB/PVA & $0.1 \%$ FL/PV A & - \\
\hline 260 & $35 \times 10^{3}$ & - & $32 \times 10^{3}$ & - \\
280 & 405 & - & 831 & - \\
300 & 373 & - & 702 & $528 \times 10^{3}$ \\
320 & 359 & $657 \times 10^{3}$ & 501 & $56 \times 10^{3}$ \\
340 & 238 & $54 \times 10^{3}$ & 328 & $16 \times 10^{3}$ \\
360 & 236 & $17 \times 10^{3}$ & 304 & $3 \times 10^{3}$ \\
380 & 233 & $3 \times 10^{3}$ & 276 & 833 \\
400 & 166 & 942 & 202 & \\
\hline
\end{tabular}



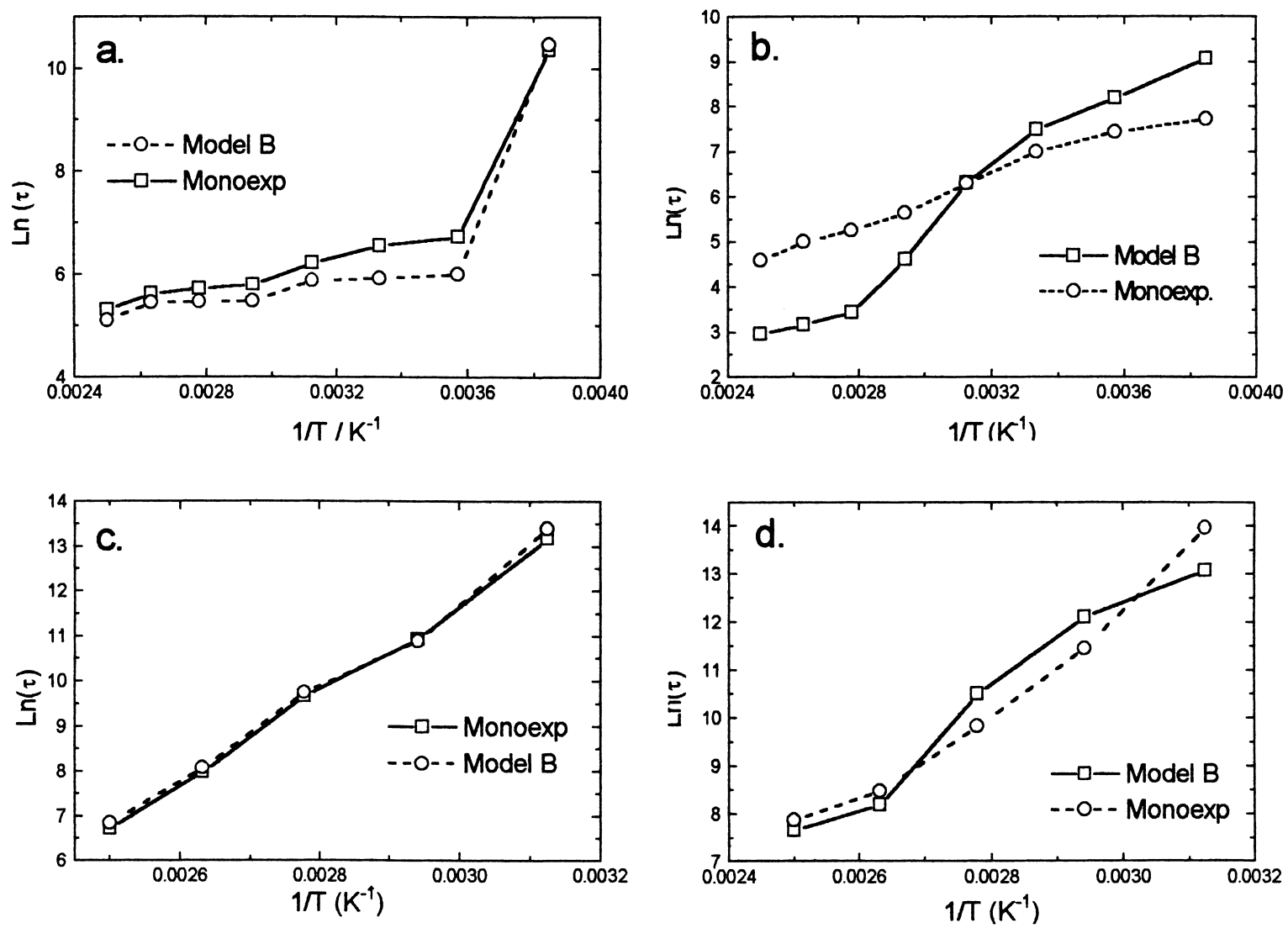

Fig. 5. Arrhenius plot for the lifetime $\left(t_{1 / \mathrm{e}}\right)$ values versus reciprocal temperatures for $0.1 \%$ FL/PVA and RB/PVA samples with compositions: $0.01 \%$ ((a) and (c)), and $0.1 \%$ ((b) and (d)), using Eq. (9) and Eq. (10).

$$
\begin{aligned}
& \mathrm{T}^{*}+\mathrm{T}^{*} \rightarrow \mathrm{DH}+\mathrm{D} \cdot\left(k_{4} \text { electron transfer }\right) \\
& \mathrm{T}^{*}+\mathrm{DH}+\mathrm{D}\left(k_{5} \text { electron transfer }\right) \\
& \mathrm{T}^{*}+\mathrm{D} \rightarrow \mathrm{S}+\mathrm{D} \cdot\left(k_{6} \text { triplet quenching by } X\right) \\
& \left.\mathrm{T}^{*}+\mathrm{DH} \cdot \mathrm{S}+\mathrm{DH} k_{7} \text { triplet quenching by } R\right) \\
& \mathrm{T}^{*}+\mathrm{O}_{2} \rightarrow \mathrm{S}+\mathrm{O}_{2}\left(k_{8} \text { physical quenching by } O_{2}\right) \\
& \mathrm{T}^{*}+\mathrm{O}_{2} \rightarrow \mathrm{D} \cdot+\mathrm{HO}_{2} \text { or } \mathrm{O}_{2}^{-}\left(k_{9} \text { chemical quenching by } O_{2}\right)
\end{aligned}
$$

where $\mathrm{S}$ is the molecule in the electronic ground state, $\mathrm{D}^{*}$ and $\mathrm{DH}^{\cdot}$ are the semioxidized and protonated semireduced molecules, respectively (Scheme 1); $\mathrm{M}=$ electron or hydrogen donor groups. In our experimental conditions both the reactions (h) and (i) were neglected since we worked in the absence of $\mathrm{O}_{2}$.<smiles>O=C1C=CC2C(=C1)Oc1cc(O)ccc1C2c1ccccc1C(=O)[O-]</smiles>

$X=D^{*}$<smiles>O=C([O-])c1ccccc1C1c2ccc(O)cc2OC2=CC([O-])C=CC21</smiles>

$\mathrm{R}=\mathrm{DH}$
Scheme 1.
Taking into account that in our experimental conditions both the reactions (h) and (i) can be neglected since we are working in the absence of $\mathrm{O}_{2}$ we are assuming that three other mechanisms must be considered in the present case: (1) The triplet quenching (reactions a-c) leading to the dye deactivation without photobleaching reaction; (2) the D--D mechanism (reactions $\mathrm{d}$ ), producing either the $\mathrm{D}^{*}$ or $\mathrm{DH}^{*}$ intermediates, and, then, the dye undergoes to its bleached form. (3) The dye photobleaching reaction in presence of either an electron or a hydrogen donor groups produce $\mathrm{D}^{*}$ or $\mathrm{DH}^{\cdot}$ (reactions e-g).

At lower concentration, the third mechanism (photobleaching in presence of either an electron or hydrogen donor groups) should be responsible by the reaction in very diluted FL/PVA samples. It is a slower process independent of the PVA relaxation processes and the kinetic can be simulated by a single-exponential function. For fluorescein at higher concentrations both elementary pathways leading to the triplet quenching and the mechanism D--D predominate should be quite effective and there was a faster photobleaching process dependent on the polymer relaxation processes. It was strongly enhanced if diffusional dye processes took place, as in the case of temperatures above the PVA glass transition. However, since at $T<T_{\mathrm{g}}$ the matrix is almost freeze, a non-diffusive mechanism must be also present indicating that a static the D--D mechanism is 
simultaneously operating at higher concentrations promoting a proximity induced $\mathrm{D}-\mathrm{-D}$ reaction. Therefore, the effectiveness of the photobleaching reaction is a weight factor between the efficiencies of the bimolecular triplettriplet deactivation and the dye-dye interaction producing a semi-reduced or semi-oxidized intermediates.

Regardless the intersystem crossing process is much more efficient for Rose Bengal than for fluorescein due to the inner heavy atom effect $[20,29]$. For example, although we do not have the rate constant for the intersystem crossing process for these dyes in PVA matrix, data reported for Rose Bengal in methanol indicate that $k_{\text {isc }}(\mathrm{RB})$ is at least two orders of magnitude faster than for fluorescein [29]. Therefore, we should expect that if the photobleaching rate constants would only be dependent on the triplet state population faster photobleaching should be obtained. Considering that the rate constants of fluorescein and Rose Bengal radiative processes are in the same time range (few nanoeconds for fluorescence and milliseconds for phosphorescence emission decays) the difference of at least 2-3 orders of magnitude for the photobleaching rates for RB/PVA compared with FL/PVA should involve subsequent slower elementary steps after the population of the triplet state. Moreover, while the photochemical rate constants for $\mathrm{RB} / \mathrm{PVA}$ is not altered by the dye concentration, differently of the FL/PVA system, the D--D mechanism (reaction d) should not be significantly operative.

In an attempt to explain the main differences between photobleaching reactivity of fluorescein and Rose Bengal we must consider not only the elementary pathways described earlier and the relative efficiencies of the bimolecular processes leading to either the triplet quenching (reactions b, c, $\mathrm{f}$ and $g$ ) or the electron transfer (reactions $d$ and e) but also the differences in the first step of the photobleaching process. Similar to fluorescein, the photobleaching process of Rose Bengal in our experimental conditions produces a colorless reaction product. However, as well established, during Rose Bengal photobleaching, there is sequential removal of halogen atoms of the xanthene moiety [9-11]. Consequently, to produce the final product, the fluorescein leuco-specie, a higher number of photons must be absorbed. Moreover, the intrinsic viscosity of the polymer matrix at $T<T_{\mathrm{g}}$ is very high that increases significantly the probability if in-cage radical recombination restoring the original unbleached dye. At present we can not demonstrate whatever is the most important competitive pathway to explain the slower photobleaching rate for Rose Bengal compared with fluorescein in PVA. There are three possible steps: (1) the necessity of a higher number of photons due to the sequential dehalogenation processes, (2) the triplet quenching process recovering the original dye molecules in the electronic ground state, and, (3) the D--D interactions produces the non-bleached molecule in the electronic ground sate. Otherwise, in the case of fluorescein the electron transfer reaction produces both the semioxidized and semireduced dye molecules and then, the D--D mechanism produces the intermediate mole- cules that are able to produce the leuco-dye. Therefore, while the D--D mechanism efficiently produces the photobleaching process of fluorescein; there is a quenching of the triplet state recovering the non-excited Rose Bengal.

\section{Conclusions}

Photobleaching kinetic of fluorescein and Rose Bengal dyes dissolved in PVA matrix were analyzed using two different approaches: the B-model and the single exponential functions. Although the B-model is not adequate to describe the kinetic rates at temperatures below the PVA glassy transition, it allowed us to calculate the average number of photons to be absorbed before the bleaching process. These data show the Rose Bengal must absorb a higher average number of photons than fluorescein, as shown by the decay constants with a higher magnitude order.

The proposed mechanism to explain the photobleaching processes for these xanthene dyes dissolved in PVA should include three types of elementary steps, involving molecules in the electronic excited triplet state. If these dyes contain halogenated xanthene moiety, another pathways related to the sequential dehalogenation steps must be included. In the absence of molecular oxygen the dye photobleaching processes in dilute samples are slow and since we did not expect bimolecular interactions between two dye molecules, the reaction should involve the PVA macromolecule. The detailed mechanism for the reaction involving the polymer chain remains unclear at the present since polymer samples do not contain extrinsic detectable additives or residual catalyst. The second type of mechanism results in a faster reaction that depends on the dye-dye interaction and predominates for higher concentrated samples. In the case of fluorescein, this process leads to an oxidized or semireduced dye form, producing the colorless di-hydro form. In the case of Rose Bengal this process quenches the triplet state producing the unbleached dye in the electronic ground state. The difference between the rate processes of the quenching and electron transfer processes of the molecules in the electronic triplet excited state should be responsible for the relative photostability of Rose Bengal in PVA matrix.

\section{Acknowledgements}

T.D.Z. Atvars thanks FAPESP and FINEP for financial support. M.T. acknowledges fellowship from FAPESP. We thank Prof. Carol Collins for useful discussions.

\section{References}

[1] G. Manivannan, R. Gangkakoti, R.A. Lessard, G. Maillot, M. Bolte, J. Phys. Chem. 97 (1993) 7228. 
[2] D.F. Eaton, Advances in Photochemistry, in: D.H. Volman, G.S. Hammond, K. Gollnick (Eds.), vol. 13, Wiley Interscience, New York, 1986, p. 427.

[3] J. Naciri, R.G. Weiss, Macromolecules 22 (1989) 3938.

[4] Z. He, G.S. Hammond, R.G. Weiss, Macromolecules 25 (1992) 502.

[5] D.C. Neckers, O.M. Valdes-Aguilera, Advances in Photochemistry, in: D. Volman, G.S. Hammond, D.C. Neckers (Eds.), vol. 18, Wiley Interscience, New York, 1993, p. 315.

[6] M. Koizumi, Y. Usui, Mol. Photochem. 4 (1972) 57.

[7] I.H. Leaver, Austr. J. Chem. 23 (1973) 753.

[8] L. Song, C.A.G.O. Varma, J.W. Verhoeven, H.J. Tanke, Biophys. J. 70 (1996) 2959.

[9] G. Oster, A.H. Adelman, J. Am. Chem. Soc., 1956, 913.

[10] G. Oster, G.K. Oster, G. Kang, J. Phys. Chem. 66 (1962) 2514.

[11] J. Paczkowski, B. Paczkowska, D.C. Neckers, J. Photochem. Photobiol. A: Chem. 61 (1991) 131.

[12] M. Talhavini, T.D.Z. Atvars, J. Photochem. Photobiol. A: Chem. 114 (1998) 65.

[13] R. Richter, Chem. Phys. Lett. 118 (1985) 534.

[14] J.C. Hoockes, J.M. Torkelson, Macromolecules 28 (1995) 7683.

[15] M. Levitus, M. Talhavini, R.M. Negri, T.D.Z. Atvars, P.F. Aramendia, J. Phys. Chem. B 101 (1997) 7680.
[16] K. Horie, I. Mita, Adv. Polym. Sci. 88 (1989) 77.

[17] L. Bokobza, Progr. Polym. Sci. 15 (1990) 337.

[18] I. Mita, K. Horie, K. Hirao, Macromolecules 22 (1989) 558.

[19] L. Song, E.J. Hennik, I.T. Young, H.J. Tanke, Biophys. J. 68 (1995) 2588.

[20] S.P. McGlynn, T. Azumi, M. Kinoshita, Molecular Spectroscopy of the Triplet State, Prentice-Hall, NJ, 1979.

[21] A. Dubois, M. Canva, A. Brun, F. Chaput, J.P. Boilot, Appl. Optics 35 (1996) 3193.

[22] D. Dibbern-Brunelli, T.D.Z. Atvars, J. Appl. Polym. Sci. 55 (1995) 889.

[23] D. Dibbern-Brunelli, T.D.Z. Atvars, J. Appl. Polym. Sci. 58 (1995) 779.

[24] M. Talhavini, T.D.Z. Atvars, Química Nova 18 (1995) 298

[25] P.D. Garrett, D.T. Grubb, J. Polym. Sci. B: Polym. Phys. 26 (1988) 2509.

[26] A. Nagai, M. Takayanagi, Rep. Progr. Polym. Sci. Japan 8 (1964) 249.

[27] L. Lindqvist, Arkiv fur Kemi 16 (1960) 817.

[28] V. Kasche, L. Lindqvist, J. Phys. Chem. 68 (1964) 817.

[29] J.B. Birks (Ed.), in: Organic Molecular Photophysics, vol. 2, Wiley, Bristol, UK, 1975, p. 153. 